CRYSTALLOGRAPHIC COMMUNICATIONS

ISSN 2056-9890

Received 11 June 2017

Accepted 7 July 2017

Edited by J. T. Mague, Tulane University, USA

Keywords: crystal structure; halogen bond; hydrogen bond; chlorodiacetylene; iododiacetylene.

CCDC references: 1551031; 1551032

Supporting information: this article has supporting information at journals.iucr.org/e

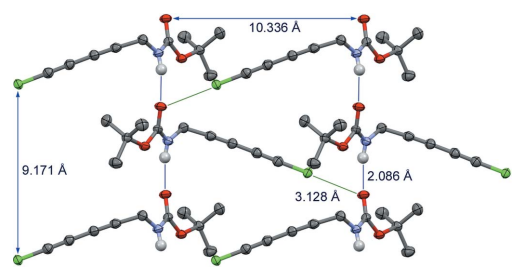

OPEN $\odot$ ACCESS

\section{Isomorphous crystal structures of chlorodiacetylene and iododiacetylene derivatives: simultaneous hydrogen and halogen bonds on carbonyl}

Pierre Baillargeon, ${ }^{\mathrm{a} *}$ Tarik Rahem, ${ }^{\mathrm{a}}$ Édouard Caron-Duval, ${ }^{\mathrm{a}}$ Jacob Tremblay, ${ }^{\mathrm{a}}$ Cloé Fortin, ${ }^{a}$ Étienne Blais, ${ }^{a}$ Victor Fan, ${ }^{a}$ Daniel Fortin ${ }^{\mathrm{b}}$ and Yves L. Dory ${ }^{\mathrm{c}}$

aDépartement de Chimie, Cégep de Sherbrooke, 475 rue du Cégep, Sherbrooke, Québec, J1E 4K1, Canada, baboratoire d'Analyses Structurales par Diffraction des Rayons-X, Département de Chimie, Université de Sherbrooke, 2500, boulevard de I'Université, Sherbrooke, Québec, J1K 2R1, Canada, and 'Laboratoire de Synthèse Supramoléculaire, Département de Chimie, Institut de Pharmacologie, Université de Sherbrooke, 3001 12e avenue nord, Sherbrooke, QC, J1H 5N4, Canada. *Correspondence e-mail: pierre.baillargeon@usherbrooke.ca

The crystal structures of tert-butyl (5-chloropenta-2,4-diyn-1-yl)carbamate, $\mathrm{C}_{10} \mathrm{H}_{12} \mathrm{ClNO}_{2}$ (II), and tert-butyl (5-iodopenta-2,4-diyn-1-yl)carbamate, $\mathrm{C}_{10} \mathrm{H}_{12} \mathrm{INO}_{2}$ (IV), are isomorphous to previously reported structures and accordingly their molecular and supramolecular structures are similar. In the crystals of (II) and (IV), molecules are linked into very similar two-dimensional wall organizations with antiparallel carbamate groups involved in a combination of hydrogen and halogen bonds (bifurcated $\mathrm{N}-\mathrm{H} \cdots \mathrm{O}=\mathrm{C}$ and $\mathrm{C} \equiv \mathrm{C}-$ $X \cdots \mathrm{O}=\mathrm{C}$ interactions on the same carbonyl group). There is no long-range parallel stacking of diynes, so the topochemical polymerization of diacetylene is prevented. A Cambridge Structural Database search revealed that $\mathrm{C} \equiv \mathrm{C}-$ $X \cdots \mathrm{O}=\mathrm{C}$ contacts shorter than the sum of the van der Waals radii are scarce (only one structure for the $\mathrm{C} \equiv \mathrm{C}-\mathrm{Cl} \cdots \mathrm{O}=\mathrm{C}$ interaction and 13 structures for the similar $\mathrm{C} \equiv \mathrm{C}-\mathrm{I} \cdots \mathrm{O}=\mathrm{C}$ interaction).

\section{Chemical context}

Hydrogen bonds (HBs) and halogen bonds (XBs) are considered to be useful noncovalent synthetic tools in crystal engineering (Aakeröy et al., 2015; Grabowski, 2016; Resnati et al., 2015; Cinčić et al., 2008). Indeed, these directional intermolecular interactions facilitate the preparation of the desired solid-state motifs and architectures (Gilday et al., 2015; Cavallo et al., 2016; Priimagi et al., 2013; Mukherjee et al., 2014; Shirman et al., 2015; Mukherjee et al., 2017). For example, using HBs and XBs, the specific organization of terminal diacetylenes (Li et al., 2009; Ouyang et al., 2003), bromodiacetylenes (Jin et al., 2015) and iododiacetylenes (Jin et al., 2013; Sun et al., 2006) has been obtained to achieve the solidstate topochemical polymerization of diacetylenes. On the other hand, to the best of our knowledge, no chlorodiacetylene topochemical polymerizations have been reported. Our results show that chlorodiacetylene (II) is isostructural to iododiacetylene (IV) and the previously reported bromodiacetylene (III) and terminal diacetylene (I) (Baillargeon et al., 2016) (see Scheme). Although the arrangement of diynes in the present article stands no chance of undergoing topochemical polymerization, we suggest that in other systems prone to polymerization, replacing $\mathrm{Br}$, I or $\mathrm{H}$ atoms by $\mathrm{Cl}$ atoms in their diyne groups might result in successful PolyChloroDiAcetylene (PCDA) formation as well. This work also contributes to an emerging research theme, namely the 
concept of orthogonal molecular interactions such as HBs and XBs (Kratzer et al., 2015; Takemura et al., 2014; Voth et al., 2009), which may find applications in medicinal chemistry and chemical biology (Wilcken et al., 2013).

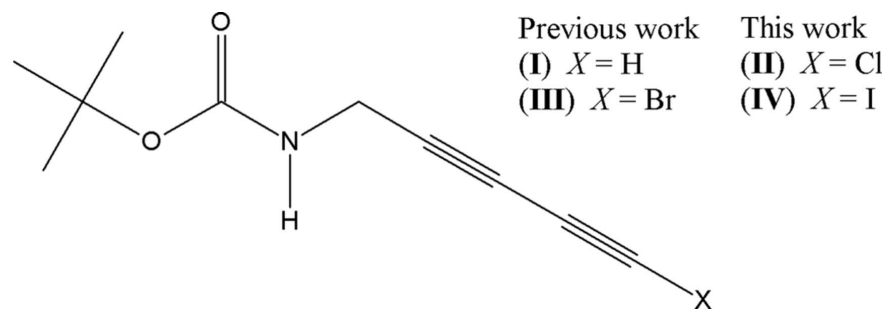

\section{Structural commentary}

The molecular structures of compounds (II) and (IV) are shown in Fig. 1. All bond lengths and angles are within normal ranges. For example, the internal diyne $\mathrm{C} 2-\mathrm{C} 3$ bonds lengths [1.376 (3) $\AA$ for (II) and 1.385 (4) $\AA$ for (IV)] follow the useful rule of thumb describing a $\mathrm{C}-\mathrm{C}$ single-bond distance $(1.54 \AA$ ) decreasing by $0.04 \AA$ each time one of the participating $\mathrm{C}$ atoms changes hybridization from $s p^{3}$ to $s p^{2}$ or from $s p^{2}$ to $s p$ (Bent, 1961). Moreover, the observed distances are almost identical to those found recently in the literature for similar halodiynes (Hoheisel et al., 2013; Baillargeon et al., 2016). The relative orientation between the diacetylenic moiety and the carbamate functional group can be established by the absolute value of the torsion angles $\mathrm{C} 4-\mathrm{C} 5-\mathrm{N} 1-\mathrm{C} 6$ [111.07 (19) $\left.{ }^{\circ}\right]$ for (II) and [103.8 (3) ${ }^{\circ}$ for (IV).

\section{Supramolecular features}

In the crystals of compounds (II) and (IV), molecules are linked via an $\mathrm{N}-\mathrm{H} \cdots \mathrm{O}=\mathrm{C}$ hydrogen bond between their

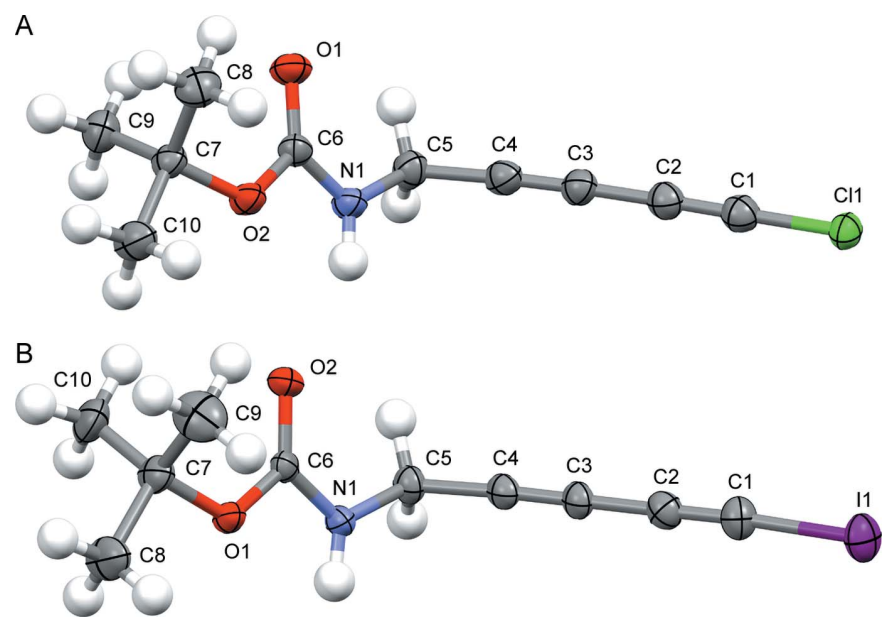

Figure 1

The molecular structure of (A) compound (II) and (B) compound (IV), showing the atom-labelling schemes. Displacement ellipsoids are drawn at the $50 \%$ probability level. $\mathrm{H}$ atoms are shown as fixed-size spheres of $0.30 \AA$ A
Table 1

Hydrogen-bond and halogen-bond geometries $\left(\AA,{ }^{\circ}\right)$ for $($ II $)$.

\begin{tabular}{lllll}
\hline$D-X \cdots A$ & $D-X$ & $X \cdots A$ & $D \cdots A$ & $D-X \cdots A$ \\
\hline $\mathrm{N} 1-\mathrm{H} 1 \cdots \mathrm{O} 1^{\mathrm{i}}$ & 0.88 & 2.09 & $2.934(2)$ & 162 \\
$\mathrm{C} 1-\mathrm{C} 11 \cdots \mathrm{O} 1^{\mathrm{ii}}$ & $1.665(2)$ & $3.127(2)$ & $4.792(3)$ & $179.01(7)$ \\
\hline
\end{tabular}

Symmetry codes: (i) $-x+2, y+\frac{1}{2},-z+\frac{1}{2}$; (ii) $-x+1, y+\frac{1}{2},-z+\frac{1}{2}$.

respective carbamate functionalities $\left[\mathrm{N} 1-\mathrm{H} 1 \cdots \mathrm{O} 1^{\mathrm{i}}\right.$ (Table 1 ) and $\mathrm{N} 1-\mathrm{H} 1 \cdots \mathrm{O} 2^{\mathrm{i}}$ (Table 2)], generating an antiparallel stacking pattern which orients the diacetylene skeleton on each side of the one-dimensional carbamate tape (parts B and $\mathrm{D}$ in Fig. 2). For both crystals, the simultaneous presence of halogen and hydrogen bonds with the carbamate $\mathrm{O}$ atom have been found. Indeed, additional halogen-bond interactions
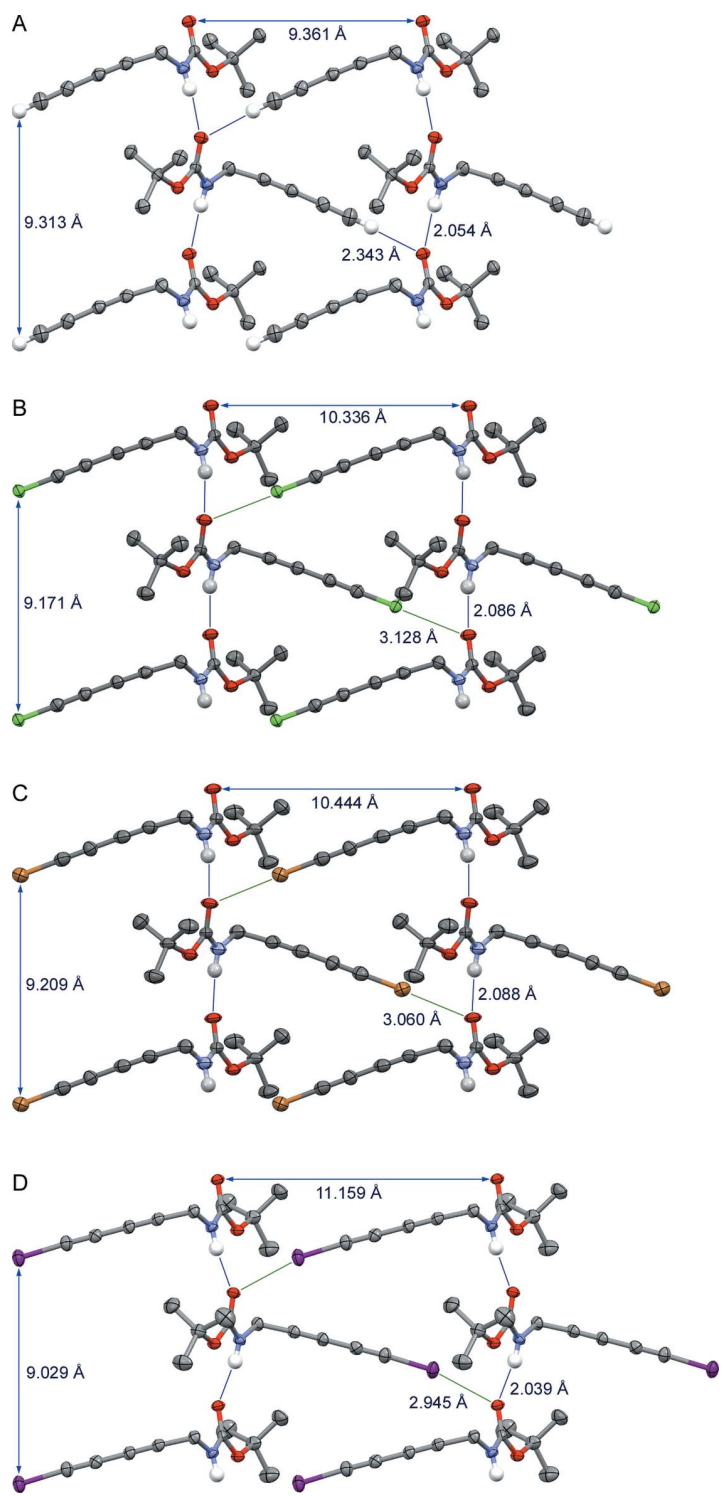

Figure 2

Halogen (green lines) and/or hydrogen bonds (blue lines) inside the supramolecular walls of (A) diyne (I), (B) chlorodiyne (II), (C) bromodiyne (III) and (D) iododiyne (IV). The nonpolar $\mathrm{H}$ atoms have been omitted for clarity. 
Table 2

Hydrogen-bond and halogen-bond geometries $\left(\AA{ }^{\circ}\right)$ for (IV).

\begin{tabular}{lllll}
\hline$D-X \cdots A$ & $D-X$ & $X \cdots A$ & $D \cdots A$ & $D-X \cdots A$ \\
\hline $\mathrm{N} 1-\mathrm{H} 1 \cdots \mathrm{O} 2^{\mathrm{i}}$ & 0.88 & 2.04 & $2.881(2)$ & 160 \\
$\mathrm{C} 1-\mathrm{I} 1 \cdots \mathrm{O} 2^{\mathrm{ii}}$ & $1.999(2)$ & $2.945(2)$ & $4.919(3)$ & $168.31(8)$ \\
\hline
\end{tabular}

Symmetry codes: (i) $-x+\frac{3}{2}, y+\frac{3}{2},-z+\frac{3}{2}$; (ii) $-x+\frac{1}{2}, y+\frac{3}{2},-z+\frac{3}{2}$.

occur with the carbamate $\mathrm{O}$ atom $\left[\mathrm{Cl} 1 \cdots \mathrm{O} 1^{\mathrm{ii}}\right.$ for $(\mathbf{I I})$ and $\mathrm{I} 1 \cdots \mathrm{O} 2^{\mathrm{ii}}$ for $\left.(\mathbf{I V})\right]$, resulting in an infinite two-dimensional network that can be considered as polar supramolecular walls. This arrangement is similar to our previous work (Baillargeon et al., 2016) on the terminal diacetylene (I) (part A in Fig. 2) and the bromodiacetylene (III) (part C in Fig. 2). In fact, diynes (I)-(IV) (Fig. 2) constitute a complete set of truly isomorphous crystals that can be carefully examined to evaluate the differences and similarities that exist between halogen and hydrogen bonds. Thus, the $X \cdots \mathrm{O} \cdots \mathrm{H}$ angle increases as the size of the halogen atom becomes larger. This angle, which is pretty open in the chlorine crystal (II) $\left(\mathrm{Cl} 1 \cdots \mathrm{O} 1 \cdots \mathrm{H} 1\right.$; part B in Fig. $\left.2 ; 69^{\circ}\right)$ adopts a near ortho-

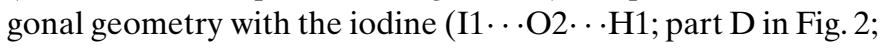
$83^{\circ}$ ). It is not a surprise that the bromine crystal (III) represents an intermediate case (part C in Fig. 2; $72^{\circ}$ ). The value for the terminal diacetylene (I) $X=\mathrm{H}$ (part A in Fig. $2 ; 76^{\circ}$ ) is closely related to the bromodiacetylene (Baillargeon et al., 2016).

\section{Database survey}

A survey of the Cambridge Structural Database (Conquest Version 1.19; CSD, Version 5.38, November 2016 plus 3 updates; Groom et al., 2016) furnished 404 hits of terminal alkynes $\mathrm{CC}-\mathrm{H}$ having close contacts with carbonyl $\mathrm{O}=\mathrm{C}$ (shorter than the sum of their van der Waals radii). On the other hand, similar contacts from halogenoalkyne analogs are scarce (1 hit for the chloroalkyne, 4 hits for the bromoalkyne and 13 hits for the iodoalkyne; Table 3). For the iodoalkyne, results are limited to monovalent iodine and for a structure in which the carbonyl group is not involved in an organometallic complex.

\section{Synthesis and crystallization}

\subsection{Compound (II)}

Tetra- $n$-butylammonium fluoride (TBAF, $0.437 \mathrm{ml}, 1 M$ in THF, $0.437 \mathrm{mmol}), \mathrm{AgNO}_{3}(39 \mathrm{mg}, 0.23 \mathrm{mmol})$ and NCS $(190 \mathrm{mg}, \quad 1.42 \mathrm{mmol})$ were added to a solution of $\mathrm{BocNHCH}_{2}-\mathrm{C} \equiv \mathrm{C}-\mathrm{C} \equiv \mathrm{C}-\mathrm{TMS}$ (183 mg, $0.728 \mathrm{mmol}$ ) in acetonitrile $(3 \mathrm{ml})$ at room temperature. The resulting mixture was stirred for $2.5 \mathrm{~h}$ under $\mathrm{N}_{2}$ in the absence of light. Purification of the crude product by flash chromatography on silica gel, eluting with mixtures of $\mathrm{Hex} / \mathrm{DCM} / \mathrm{Et}_{2} \mathrm{O}$ (gradient from 9:1:1 to 1:1:1), provided compound (II) as a beige solid (yield $72 \mathrm{mg}, 46 \%$ ). Single crystals suitable for X-ray diffraction were prepared by diffusion of pentane into a chloroform solution of (II) at $263 \mathrm{~K} . R_{\mathrm{F}}=0.43\left(2: 1: 1 \mathrm{Hex} / \mathrm{DCM} / \mathrm{Et}_{2} \mathrm{O}\right)$; IR (UATR, $v, \mathrm{~cm}^{-1}$ ): 3326, 2977, 2920, 2255, 2168, 1673, 1531, 1421, 1368, 1278, 1248, 1222, 1158, 1143, 1042, 1028, 933, 849, 761, 718, 655; ${ }^{1} \mathrm{H}$ NMR $\left(400 \mathrm{MHz}, \mathrm{CDCl}_{3}\right): \delta 4.72(b r, 1 \mathrm{H})$, $3.99(d, 2 \mathrm{H}), 1.45(\mathrm{~s}, 9 \mathrm{H})$; HRMS $(\mathrm{m} / \mathrm{z})$ : calculated for $\mathrm{C}_{10} \mathrm{H}_{12} \mathrm{ClNNaO}_{2}\left[\mathrm{MNa}^{+}\right]: 236.0449$, found: 236.0448 .

\subsection{Compound (IV)}

TBAF (0.437 ml, $1 M$ in THF, $0.437 \mathrm{mmol}), \mathrm{AgNO}_{3}(39 \mathrm{mg}$, $0.23 \mathrm{mmol}$ ) and NIS (328 $\mathrm{mg}, 1.46 \mathrm{mmol}$ ) were added to a solution of $\mathrm{BocNHCH}_{2}-\mathrm{C} \equiv \mathrm{C}-\mathrm{C} \equiv \mathrm{C}-\mathrm{TMS} \quad(183 \mathrm{mg}$, $0.728 \mathrm{mmol})$ in acetonitrile $(3 \mathrm{ml})$ at room temperature. The resulting mixture was stirred for $2.5 \mathrm{~h}$ under $\mathrm{N}_{2}$ in the absence of light. Purification of the crude product by flash chromatography on silica gel, eluting with mixtures of $\mathrm{Hex} / \mathrm{DCM} / \mathrm{Et}_{2} \mathrm{O}$ (gradient from 9:1:1 to 1:1:1) provided compound (IV) as a beige solid (yield $95 \mathrm{mg}, 43 \%$ ). Single crystals suitable for

Table 3

CSD data (Groom et al., 2016) retrieved for the $\mathrm{C} \equiv \mathrm{C}-X \cdots \mathrm{O}=\mathrm{C}$ contacts shorter than the sum of their van der Waals radii.

\begin{tabular}{|c|c|c|c|c|c|}
\hline $\mathrm{C} \equiv \mathrm{C}-X \cdots \mathrm{O}=\mathrm{C}$ contacts & CSD refcode & Space group & $X \ldots \mathrm{O}$ distance $(\AA)$ & $\mathrm{C}-X \cdots \mathrm{O}$ angle $\left(^{\circ}\right)$ & Reference \\
\hline $\mathrm{C} \equiv \mathrm{C}-\mathrm{Br} \cdots \mathrm{O}=\mathrm{C}$ & HEVWAI & $C 2$ & 2.959 & 158.12 & Hoheisel et al. (2013) \\
\hline $\mathrm{C} \equiv \mathrm{C}-\mathrm{Br} \cdots \mathrm{O}=\mathrm{C}$ & HEVWAI01 & $P 2_{1} 2_{1} 2_{1}$ & 2.966 & 166.70 & Hoheisel et al. (2013) \\
\hline $\mathrm{C} \equiv \mathrm{C}-\mathrm{Br} \cdots \mathrm{O}=\mathrm{C}$ & KAMXII & $P 2_{1} / c$ & 3.060 & 178.26 & Baillargeon et al. (2016) \\
\hline $\mathrm{C} \equiv \mathrm{C}-\mathrm{I} \cdots \mathrm{O}=\mathrm{C}$ & COHYUU & $P \overline{1}$ & 3.096 & 164.55 & Luo et al. (2008) \\
\hline $\mathrm{C} \equiv \mathrm{C}-\mathrm{I} \cdots \mathrm{O}=\mathrm{C}$ & IYAYUC & Pca2 $2_{1}$ & 2.861 & 170.36 & Hou et al. (2004) \\
\hline $\mathrm{C} \equiv \mathrm{C}-\mathrm{I} \cdots \mathrm{O}=\mathrm{C}$ & LUNKOW & $P 2 / c$ & 2.791 & 174.12 & Kratzer et al. (2015) \\
\hline $\mathrm{C} \equiv \mathrm{C}-\mathrm{I} \cdots \mathrm{O}=\mathrm{C}$ & LUNKUC & $P 2_{1} / c$ & 2.754 & 172.63 & Kratzer et al. (2015) \\
\hline $\mathrm{C} \equiv \mathrm{C}-\mathrm{I} \cdots \mathrm{O}=\mathrm{C}$ & LUNLAJ & $P 2_{1} / c$ & 2.773 & 173.70 & Kratzer et al. (2015) \\
\hline $\mathrm{C} \equiv \mathrm{C}-\mathrm{I} \cdots \mathrm{O}=\mathrm{C}$ & LUNLIR & Pca2 $_{1}$ & 2.858 & 170.94 & Kratzer et al. (2015) \\
\hline $\mathrm{C} \equiv \mathrm{C}-\mathrm{I} \cdots \mathrm{O}=\mathrm{C}$ & LUNLOX & $C 2 / c$ & 2.763 & 175.58 & Kratzer et al. (2015) \\
\hline $\mathrm{C} \equiv \mathrm{C}-\mathrm{I} \cdots \mathrm{O}=\mathrm{C}$ & IBUYAI & $P 2_{1} / m$ & 2.856 & 177.96 & Dumele et al. (2017) \\
\hline
\end{tabular}


Table 4

Experimental details.

(II)

Crystal data

Chemical formula

$M_{\mathrm{r}}$

Crystal system, space group

Temperature $(\mathrm{K})$

$a, b, c(\AA)$

$\beta\left({ }^{\circ}\right)$

$V\left(\AA^{3}\right)$

Z

Radiation type

$\mu\left(\mathrm{mm}^{-1}\right)$

Crystal size (mm)

Data collection

Diffractometer

Absorption correction

$T_{\min }, T_{\max }$

No. of measured, independent and observed

$[I>2 \sigma(I)]$ reflections

$R_{\text {int }}$

$(\sin \theta / \lambda)_{\max }\left(\AA^{-1}\right)$

Refinement

$R\left[F^{2}>2 \sigma\left(F^{2}\right)\right], w R\left(F^{2}\right), S$

No. of reflections

No. of parameters

$\mathrm{H}$-atom treatment

$\Delta \rho_{\max }, \Delta \rho_{\min }\left(\mathrm{e} \AA^{-3}\right)$
(IV)

$\mathrm{C}_{10} \mathrm{H}_{12} \mathrm{ClNO}_{2}$
213.66
Monoclinic, $P 2_{1} / c$
173
$10.336(3), 9.171(3), 11.870(3)$
$100.656(5)$
$1105.8(5)$
4
Mo $K \alpha$
0.32
$0.34 \times 0.22 \times 0.02$

$\mathrm{C}_{10} \mathrm{H}_{12} \mathrm{INO}_{2}$

305.11

Monoclinic, $P 2_{1} / n$

173

11.1587 (16), 9.0288 (13), 12.9899 (18)

108.731 (2)

1239.4 (3)

4

Mo $K \alpha$

2.56

$0.36 \times 0.3 \times 0.28$

Bruker APEXII

Multi-scan (SADABS; Bruker, 2008)

$0.66,0.745$

16132, 2249, 1755

0.045

0.625

Bruker APEXII

Multi-scan (SADABS; Bruker, 2008) $0.675,0.745$

17970, 2532, 2342

0.02

0.626

$0.036,0.089,1.06$
2249
130
H-atom parameters constrained
$0.22,-0.21$

0.022, 0.054, 1.08

2532

130

H-atom parameters constrained $1.32,-0.69$

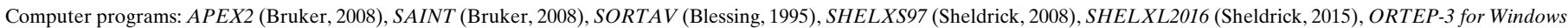
(Farrugia, 2012), Mercury (Macrae et al., 2006), Win GX (Farrugia, 2012) and publCIF (Westrip, 2010).

X-ray diffraction were prepared by slow evaporation from a chloroform solution of $(\mathbf{I V})$ at room temperature. $R_{\mathrm{F}}=0.48$ (2:1:1 Hex/DCM/Et $\left.{ }_{2} \mathrm{O}\right)$; IR (UATR, $\left.v, \mathrm{~cm}^{-1}\right): 3328,2980$, 2933, 2230, 2159, 1661, 1532, 1451, 1420, 1367, 1284, 1250, 1154, 1142, 1042, 1026, 929, 851, 762, 714, 647; ${ }^{1} \mathrm{H}$ NMR (400 MHz, $\left.\mathrm{CDCl}_{3}\right): \delta 4.73(b r, 1 \mathrm{H}), 4.02(d, 2 \mathrm{H}), 1.44(s, 9 \mathrm{H})$.

\section{Refinement}

Crystal data, data collection and structure refinement details are summarized in Table 4.

\section{Funding information}

Funding for this research was provided by: Fonds de Recherche du Québec - Nature et Technologies (grant No. 2016-CO-194882); Centre d'étude et de recherche transdisciplinaire étudiants-enseignants (CERTEE, Cégep de Sherbrooke); Fondation du Cégep de Sherbrooke.

\section{References}

Aakeröy, C. B., Spartz, C. L., Dembowski, S., Dwyre, S. \& Desper, J. (2015). IUCrJ, 2, 498-510.

Avtomonov, E. V., Grüning, R. \& Lorberth, J. (1997). Z. Naturforsch. Teil B, 52, 256-258.

Baillargeon, P., Caron-Duval, É., Pellerin, É., Gagné, S. \& Dory, Y. L. (2016). Crystals, 6, 37-49.

Bent, H. A. (1961). Chem. Rev. 61, 275-311.
Blessing, R. H. (1995). Acta Cryst. A51, 33-38.

Bruker (2008). APEX2, SAINT and SADABS. Bruker AXS Inc., Madison, Wisconsin, USA.

Cavallo, G., Metrangolo, P., Milani, R., Pilati, T., Priimagi, A., Resnati, G. \& Terraneo, G. (2016). Chem. Rev. 116, 2478-2601.

Cinčić, D., Friščić, T. \& Jones, W. (2008). Chem. Mater. 20, 66236626.

Dumele, O., Schreib, B., Warzok, U., Trapp, N., Schalley, C. A. \& Diederich, F. (2017). Angew. Chem. Int. Ed. 56, 1152-1157.

Dumele, O., Wu, D., Trapp, N., Goroff, N. \& Diederich, F. (2014). Org. Lett. 16, 4722-4725.

Farrugia, L. J. (2012). J. Appl. Cryst. 45, 849-854.

Gilday, L. C., Robinson, S. W., Barendt, T. A., Langton, M. J., Mullaney, B. R. \& Beer, P. D. (2015). Chem. Rev. 115, 7118-7195.

Grabowski, S. J. (2016). Crystals, 6, 59-63.

Groom, C. R., Bruno, I. J., Lightfoot, M. P. \& Ward, S. C. (2016). Acta Cryst. B72, 171-179.

Hoheisel, T. N., Schrettl, S., Marty, R., Todorova, T. K., Corminboeuf, C. \& Sienkiewicz, A. (2013). Nat. Chem. 5, 327-334.

Hou, Z.-K., Ren, Y.-G., Huang, M.-Z., Song, J. \& Chen, L.-G. (2004). Acta Cryst. E60, o1336-o1337.

Jin, H., Plonka, A. M., Parise, J. B. \& Goroff, N. S. (2013). CrystEngComm, 15, 3106-3110.

Jin, H., Young, C. N., Halada, G. P., Phillips, B. L. \& Goroff, N. S. (2015). Angew. Chem. Int. Ed. 54, 14690-14695.

Kawai, H., Utamura, T., Motoi, E., Takahashi, T., Sugino, H., Tamura, M., Ohkita, M., Fujiwara, K., Saito, T., Tsuji, T. \& Suzuki, T. (2013). Chem. Eur. J. 19, 4513-4524.

Kratzer, P., Ramming, B., Römisch, S. \& Maas, G. (2015). CrystEngComm, 17, 4486-4494.

Li, Z., Fowler, F. W. \& Lauher, J. W. (2009). J. Am. Chem. Soc. 131, 634-643.

Luo, L., Wilhelm, C., Sun, A., Grey, C. P., Lauher, J. W. \& Goroff, N. S. (2008). J. Am. Chem. Soc. 130, 7702-7709. 
Macrae, C. F., Edgington, P. R., McCabe, P., Pidcock, E., Shields, G. P., Taylor, R., Towler, M. \& van de Streek, J. (2006). J. Appl. Cryst. 39, $453-457$.

Mukherjee, A., Teyssandier, J., Hennrich, G., De Feyter, S. \& Mali, K. S. (2017). Chem. Sci. 8, 3759-3769.

Mukherjee, A., Tothadi, S. \& Desiraju, G. R. (2014). Acc. Chem. Res. 47, 2514-2524.

Ouyang, X., Fowler, F. W. \& Lauher, J. W. (2003). J. Am. Chem. Soc. 125, 12400-12401.

Perkins, C., Libri, S., Adams, H. \& Brammer, L. (2012). CrystEngComm, 14, 3033-3038.

Priimagi, A., Cavallo, G., Metrangolo, P. \& Restani, R. (2013). Acc. Chem. Res. 46, 2686-2695.

Resnati, G., Boldyreva, E., Bombicz, P. \& Kawano, M. (2015). IUCrJ, 2, 675-690.
Sheldrick, G. M. (2008). Acta Cryst. A64, 112-122.

Sheldrick, G. M. (2015). Acta Cryst. C71, 3-8.

Shirman, T., Boterashvili, M., Orbach, M., Freeman, D., Shimon, L. J. W., Lahav, M. \& van der Boom, M. E. (2015). Cryst. Growth Des. 15, 4756-4759.

Sun, A., Lauher, J. W. \& Goroff, N. S. (2006). Science, 312, 1030-1034. Takemura, A., McAllister, L. J., Hart, S., Pridmore, N. E., Karadakov, P. B., Whitwood, A. C. \& Bruce, D. W. (2014). Chem. Eur. J. 20, 6721-6732.

Voth, A. R., Khuu, P., Oishi, K. \& Ho, P. S. (2009). Nat. Chem. 1, 7479.

Westrip, S. P. (2010). J. Appl. Cryst. 43, 920-925.

Wilcken, R., Zimmermann, M. O., Lange, A., Joerger, A. C. \& Boeckler, F. M. (2013). J. Med. Chem. 56, 1363-1388. 


\section{supporting information}

Acta Cryst. (2017). E73, 1175-1179 [https://doi.org/10.1107/S2056989017010155]

Isomorphous crystal structures of chlorodiacetylene and iododiacetylene derivatives: simultaneous hydrogen and halogen bonds on carbonyl

Pierre Baillargeon, Tarik Rahem, Édouard Caron-Duval, Jacob Tremblay, Cloé Fortin, Étienne Blais, Victor Fan, Daniel Fortin and Yves L. Dory

Computing details

For both structures, data collection: APEX2 (Bruker, 2008); cell refinement: SAINT (Bruker, 2008); data reduction: SORTAV (Blessing, 1995); program(s) used to solve structure: SHELXS97 (Sheldrick, 2008); program(s) used to refine structure: SHELXL2016 (Sheldrick, 2015); molecular graphics: ORTEP-3 for Windows (Farrugia, 2012) and Mercury (Macrae et al., 2006); software used to prepare material for publication: WinGX (Farrugia, 2012) and publCIF (Westrip, 2010).

tert-Butyl (5-chloropenta-2,4-diyn-1-yl)carbamate (II)

Crystal data

$\mathrm{C}_{10} \mathrm{H}_{12} \mathrm{ClNO}_{2}$

$M_{r}=213.66$

Monoclinic, $P 2_{1} / c$

Hall symbol: -P $2 \mathrm{ybc}$

$a=10.336(3) \AA$

$b=9.171(3) \AA$

$c=11.870(3) \AA$

$\beta=100.656(5)^{\circ}$

$V=1105.8(5) \AA^{3}$

$Z=4$

$F(000)=448$

$D_{\mathrm{x}}=1.283 \mathrm{Mg} \mathrm{m}^{-3}$

Mo $K \alpha$ radiation, $\lambda=0.71073 \AA$

Cell parameters from 8841 reflections

$\theta=2.8-26.4^{\circ}$

$\mu=0.32 \mathrm{~mm}^{-1}$

$T=173 \mathrm{~K}$

Plate, orange

$0.34 \times 0.22 \times 0.02 \mathrm{~mm}$

\section{Data collection}

Bruker APEXII

diffractometer

Radiation source: sealed x-ray tube Graphite monochromator

$\varphi$ or $\omega$ oscillation scans

Absorption correction: multi-scan

(SADABS; Bruker, 2008)

$T_{\text {min }}=0.66, T_{\text {max }}=0.745$

16132 measured reflections

2249 independent reflections

1755 reflections with $I>2 \sigma(I)$

$R_{\text {int }}=0.045$

$\theta_{\text {max }}=26.4^{\circ}, \theta_{\min }=2.0^{\circ}$

$h=-12 \rightarrow 12$

$k=-11 \rightarrow 11$

$l=-9 \rightarrow 14$

\section{Refinement}

Refinement on $F^{2}$

Least-squares matrix: full

$R\left[F^{2}>2 \sigma\left(F^{2}\right)\right]=0.036$

$w R\left(F^{2}\right)=0.089$

$S=1.06$

2249 reflections

130 parameters

0 restraints

0 constraints

Hydrogen site location: inferred from

neighbouring sites

$\mathrm{H}$-atom parameters constrained 
$w=1 /\left[\sigma^{2}\left(F_{\mathrm{o}}^{2}\right)+(0.0397 P)^{2}+0.2863 P\right]$

where $P=\left(F_{\mathrm{o}}^{2}+2 F_{\mathrm{c}}^{2}\right) / 3$

$(\Delta / \sigma)_{\max }<0.001$

$$
\Delta \rho_{\max }=0.22 \text { e } \AA^{-3}
$$

Special details

Geometry. All esds (except the esd in the dihedral angle between two 1.s. planes) are estimated using the full covariance matrix. The cell esds are taken into account individually in the estimation of esds in distances, angles and torsion angles; correlations between esds in cell parameters are only used when they are defined by crystal symmetry. An approximate (isotropic) treatment of cell esds is used for estimating esds involving 1.s. planes.

Fractional atomic coordinates and isotropic or equivalent isotropic displacement parameters $\left(\AA^{2}\right)$

\begin{tabular}{lllll}
\hline & $x$ & $y$ & $z$ & $U_{\text {iso }} * U_{\text {eq }}$ \\
\hline C1 & $0.40035(19)$ & $0.0211(2)$ & $0.15762(16)$ & $0.0343(4)$ \\
C2 & $0.50716(18)$ & $-0.0266(2)$ & $0.15615(16)$ & $0.0336(4)$ \\
C3 & $0.63096(19)$ & $-0.0798(2)$ & $0.15309(16)$ & $0.0336(4)$ \\
C4 & $0.73990(19)$ & $-0.1223(2)$ & $0.15037(16)$ & $0.0334(4)$ \\
C5 & $0.87519(17)$ & $-0.1687(2)$ & $0.14761(17)$ & $0.0339(4)$ \\
H5A & 0.882935 & -0.274828 & 0.16243 & $0.041^{*}$ \\
H5B & 0.894345 & -0.150569 & 0.070125 & $0.041^{*}$ \\
C6 & $1.04144(17)$ & $-0.15939(19)$ & $0.32333(15)$ & $0.0271(4)$ \\
C7 & $1.22986(18)$ & $-0.11065(19)$ & $0.47967(15)$ & $0.0304(4)$ \\
C8 & $1.1748(2)$ & $-0.1841(2)$ & $0.57483(17)$ & $0.0439(5)$ \\
H8A & 1.106364 & -0.122368 & 0.597047 & $0.066^{*}$ \\
H8B & 1.245644 & -0.198955 & 0.641132 & $0.066^{*}$ \\
H8C & 1.136913 & -0.278618 & 0.547923 & $0.066^{*}$ \\
C9 & $1.32333(19)$ & $-0.2075(2)$ & $0.42986(18)$ & $0.0408(5)$ \\
H9A & 1.278745 & -0.298815 & 0.40299 & $0.061^{*}$ \\
H9B & 1.400577 & -0.228971 & 0.488902 & $0.061^{*}$ \\
H9C & 1.351283 & -0.15766 & 0.365371 & $0.061^{*}$ \\
C10 & $1.2953(2)$ & $0.0332(2)$ & $0.52078(19)$ & $0.0449(5)$ \\
H10A & 1.324136 & 0.082715 & 0.456527 & $0.067^{*}$ \\
H10B & 1.371735 & 0.014414 & 0.581378 & $0.067^{*}$ \\
H10C & 1.232365 & 0.095115 & 0.550916 & $0.067^{*}$ \\
C11 & $0.24981(4)$ & $0.08590(5)$ & $0.15756(4)$ & $0.03061(14)$ \\
N1 & $0.97160(14)$ & $-0.09282(16)$ & $0.23144(13)$ & $0.0314(4)$ \\
H1 & 0.984766 & 0.000739 & 0.221551 & $0.038^{*}$ \\
O1 & $1.03225(13)$ & $-0.28874(13)$ & $0.34594(11)$ & $0.0361(3)$ \\
O2 & $1.12149(12)$ & $-0.06256(12)$ & $0.38708(11)$ & $0.0311(3)$ \\
& & & & \\
& & & &
\end{tabular}

Atomic displacement parameters $\left(\AA^{2}\right)$

\begin{tabular}{lllllll}
\hline & $U^{11}$ & $U^{22}$ & $U^{33}$ & $U^{12}$ & $U^{13}$ & $U^{23}$ \\
\hline C1 & $0.0342(11)$ & $0.0349(11)$ & $0.0334(11)$ & $-0.0009(9)$ & $0.0055(8)$ & $0.0009(8)$ \\
C2 & $0.0337(11)$ & $0.0333(10)$ & $0.0323(11)$ & $-0.0036(8)$ & $0.0019(8)$ & $0.0007(8)$ \\
C3 & $0.0333(11)$ & $0.0300(10)$ & $0.0346(11)$ & $-0.0005(8)$ & $-0.0011(8)$ & $0.0016(8)$ \\
C4 & $0.0339(11)$ & $0.0279(10)$ & $0.0350(11)$ & $-0.0023(8)$ & $-0.0028(8)$ & $-0.0006(8)$ \\
C5 & $0.0309(10)$ & $0.0311(10)$ & $0.0368(11)$ & $0.0013(8)$ & $-0.0014(8)$ & $-0.0030(8)$ \\
C6 & $0.0264(9)$ & $0.0222(9)$ & $0.0323(10)$ & $-0.0014(7)$ & $0.0045(7)$ & $-0.0023(7)$
\end{tabular}


supporting information

\begin{tabular}{lllllll} 
C7 & $0.0311(10)$ & $0.0269(10)$ & $0.0299(10)$ & $0.0017(8)$ & $-0.0026(8)$ & $0.0021(8)$ \\
C8 & $0.0524(13)$ & $0.0429(12)$ & $0.0369(12)$ & $0.0014(10)$ & $0.0091(10)$ & $0.0049(9)$ \\
C9 & $0.0344(11)$ & $0.0415(12)$ & $0.0451(13)$ & $0.0056(9)$ & $0.0037(9)$ & $0.0019(9)$ \\
C10 & $0.0468(13)$ & $0.0339(11)$ & $0.0462(13)$ & $-0.0041(10)$ & $-0.0121(10)$ & $-0.0007(9)$ \\
C11 & $0.0268(2)$ & $0.0320(3)$ & $0.0337(3)$ & $0.00451(19)$ & $0.00733(18)$ & $0.00090(19)$ \\
N1 & $0.0303(8)$ & $0.0220(8)$ & $0.0381(9)$ & $-0.0024(7)$ & $-0.0036(7)$ & $0.0013(7)$ \\
O1 & $0.0409(8)$ & $0.0217(7)$ & $0.0427(8)$ & $-0.0034(6)$ & $0.0003(6)$ & $0.0014(6)$ \\
O2 & $0.0314(7)$ & $0.0220(7)$ & $0.0358(8)$ & $-0.0009(5)$ & $-0.0049(6)$ & $-0.0006(5)$ \\
\hline
\end{tabular}

Geometric parameters $\left(\AA,{ }^{\circ}\right)$

\begin{tabular}{llll}
\hline $\mathrm{C} 1-\mathrm{C} 2$ & $1.191(3)$ & $\mathrm{C} 6-\mathrm{N} 1$ & $1.339(2)$ \\
$\mathrm{C} 1-\mathrm{C} 11$ & $1.666(2)$ & $\mathrm{C} 6-\mathrm{O} 2$ & $1.347(2)$ \\
$\mathrm{C} 2-\mathrm{C} 3$ & $1.376(3)$ & $\mathrm{C} 7-\mathrm{O} 2$ & $1.484(2)$ \\
$\mathrm{C} 3-\mathrm{C} 4$ & $1.198(3)$ & $\mathrm{C} 7-\mathrm{C} 9$ & $1.511(3)$ \\
$\mathrm{C} 4-\mathrm{C} 5$ & $1.468(3)$ & $\mathrm{C} 7-\mathrm{C} 8$ & $1.513(3)$ \\
$\mathrm{C} 5-\mathrm{N} 1$ & $1.449(2)$ & $\mathrm{C} 7-\mathrm{C} 10$ & $1.521(3)$ \\
$\mathrm{C} 6-\mathrm{O} 1$ & $1.224(2)$ & & \\
& & & $109.55(15)$ \\
$\mathrm{C} 2-\mathrm{C} 1-\mathrm{C} 11$ & $178.9(2)$ & $\mathrm{O} 2-\mathrm{C} 7-\mathrm{C} 9$ & $110.41(15)$ \\
$\mathrm{C} 1-\mathrm{C} 2-\mathrm{C} 3$ & $179.0(2)$ & $\mathrm{O} 2-\mathrm{C} 7-\mathrm{C} 8$ & $112.70(16)$ \\
$\mathrm{C} 4-\mathrm{C} 3-\mathrm{C} 2$ & $178.2(2)$ & $\mathrm{C}-\mathrm{C} 7-\mathrm{C} 8$ & $102.12(14)$ \\
$\mathrm{C} 3-\mathrm{C} 4-\mathrm{C} 5$ & $177.8(2)$ & $\mathrm{C} 9-\mathrm{C} 7-\mathrm{C} 10$ & $110.89(17)$ \\
$\mathrm{N} 1-\mathrm{C} 5-\mathrm{C} 4$ & $112.49(16)$ & $\mathrm{C} 8-\mathrm{C} 7-\mathrm{C} 10$ & $122.64(17)$ \\
$\mathrm{O} 1-\mathrm{C} 6-\mathrm{N} 1$ & $124.66(17)$ & $\mathrm{C} 6-\mathrm{N} 1-\mathrm{C} 5$ & $121.42(13)$ \\
$\mathrm{O} 1-\mathrm{C} 6-\mathrm{O} 2$ & $125.48(17)$ & $\mathrm{C}-\mathrm{O} 2-\mathrm{C} 7$ & $-166.78(14)$ \\
$\mathrm{N} 1-\mathrm{C} 6-\mathrm{O} 2$ & $109.86(15)$ & $\mathrm{N} 1-\mathrm{C} 6-\mathrm{O} 2-\mathrm{C} 7$ & $59.3(2)$ \\
& & $\mathrm{C} 9-\mathrm{C} 7-\mathrm{O} 2-\mathrm{C} 6$ & $-65.4(2)$ \\
$\mathrm{O} 1-\mathrm{C} 6-\mathrm{N} 1-\mathrm{C} 5$ & $0.3(3)$ & $\mathrm{C} 10-\mathrm{C} 7-\mathrm{O} 2-\mathrm{C} 6$ & $176.85(16)$ \\
$\mathrm{O} 2-\mathrm{C} 6-\mathrm{N} 1-\mathrm{C} 5$ & $-178.98(15)$ & & \\
$\mathrm{C} 4-\mathrm{C} 5-\mathrm{N} 1-\mathrm{C} 6$ & $111.1(2)$ & & \\
$\mathrm{O} 1-\mathrm{C} 6-\mathrm{O} 2-\mathrm{C} 7$ & $13.9(3)$ & &
\end{tabular}

Hydrogen-bond geometry $\left(\AA,{ }^{\circ}\right)$

\begin{tabular}{lllll}
\hline$D-\mathrm{H} \cdots A$ & $D-\mathrm{H}$ & $\mathrm{H} \cdots A$ & $D \cdots A$ & $D-\mathrm{H} \cdots A$ \\
\hline $\mathrm{N} 1-\mathrm{H} 1 \cdots \mathrm{O} 1^{\mathrm{i}}$ & 0.88 & 2.09 & 2.935 & 162 \\
$\mathrm{C} 1-\mathrm{Cl1} \cdots \mathrm{O} 1^{\mathrm{ii}}$ & 1.67 & 3.13 & 4.793 & 179 \\
\hline
\end{tabular}

Symmetry codes: (i) $-x+2, y+1 / 2,-z+1 / 2$; (ii) $-x+1, y+1 / 2,-z+1 / 2$.

tert-Butyl (5-iodopenta-2,4-diyn-1-yl)carbamate (IV)

Crystal data

$\mathrm{C}_{10} \mathrm{H}_{12} \mathrm{INO}_{2}$

$M_{r}=305.11$

Monoclinic, $P 2_{1} / n$

Hall symbol: -P 2 yn

$a=11.1587$ (16) $\AA$

$b=9.0288(13) \AA$

$$
\begin{aligned}
& c=12.9899(18) \AA \\
& \beta=108.731(2)^{\circ} \\
& V=1239.4(3) \AA^{3} \\
& Z=4 \\
& F(000)=592 \\
& D_{\mathrm{x}}=1.635 \mathrm{Mg} \mathrm{m}^{-3}
\end{aligned}
$$


Mo $K \alpha$ radiation, $\lambda=0.71073 \AA$

Cell parameters from 9940 reflections

$\theta=2.3-26.4^{\circ}$

$\mu=2.56 \mathrm{~mm}^{-1}$

\section{Data collection}

Bruker APEXII

diffractometer

Radiation source: sealed x-ray tube

Graphite monochromator

$\varphi$ or $\omega$ oscillation scans

Absorption correction: multi-scan

(SADABS; Bruker, 2008)

$T_{\min }=0.675, T_{\max }=0.745$

\section{Refinement}

Refinement on $F^{2}$

Least-squares matrix: full

$R\left[F^{2}>2 \sigma\left(F^{2}\right)\right]=0.022$

$w R\left(F^{2}\right)=0.054$

$S=1.08$

2532 reflections

130 parameters

0 restraints

0 constraints
$T=173 \mathrm{~K}$

Prism, yellow

$0.36 \times 0.3 \times 0.28 \mathrm{~mm}$

17970 measured reflections

2532 independent reflections

2342 reflections with $I>2 \sigma(I)$

$R_{\text {int }}=0.02$

$\theta_{\text {max }}=26.4^{\circ}, \theta_{\min }=2.1^{\circ}$

$h=-12 \rightarrow 13$

$k=-11 \rightarrow 11$

$l=-15 \rightarrow 16$

Hydrogen site location: inferred from neighbouring sites

$\mathrm{H}$-atom parameters constrained

$w=1 /\left[\sigma^{2}\left(F_{\mathrm{o}}^{2}\right)+(0.0203 P)^{2}+1.7447 P\right]$

where $P=\left(F_{\mathrm{o}}^{2}+2 F_{\mathrm{c}}^{2}\right) / 3$

$(\Delta / \sigma)_{\max }=0.001$

$\Delta \rho_{\max }=1.32 \mathrm{e} \AA^{-3}$

$\Delta \rho_{\min }=-0.69$ e $\AA^{-3}$

Special details

Geometry. All esds (except the esd in the dihedral angle between two 1.s. planes) are estimated using the full covariance matrix. The cell esds are taken into account individually in the estimation of esds in distances, angles and torsion angles; correlations between esds in cell parameters are only used when they are defined by crystal symmetry. An approximate (isotropic) treatment of cell esds is used for estimating esds involving l.s. planes.

Fractional atomic coordinates and isotropic or equivalent isotropic displacement parameters $\left(\AA^{2}\right)$

\begin{tabular}{lllll}
\hline & $x$ & $y$ & $z$ & $U_{\text {iso }} * / U_{\text {eq }}$ \\
\hline C1 & $0.2157(2)$ & $1.0340(3)$ & $0.3437(2)$ & $0.0328(6)$ \\
C2 & $0.3210(2)$ & $1.0012(3)$ & $0.34873(19)$ & $0.0304(5)$ \\
C3 & $0.4425(2)$ & $0.9623(3)$ & $0.35280(19)$ & $0.0279(5)$ \\
C4 & $0.5465(2)$ & $0.9286(3)$ & $0.35525(19)$ & $0.0275(5)$ \\
C5 & $0.6760(2)$ & $0.8938(3)$ & $0.35692(19)$ & $0.0282(5)$ \\
H5A & 0.736704 & 0.927089 & 0.42691 & $0.034^{*}$ \\
H5B & 0.684833 & 0.785076 & 0.352172 & $0.034^{*}$ \\
C6 & $0.7111(2)$ & $0.8882(2)$ & $0.18051(19)$ & $0.0219(4)$ \\
C7 & $0.7579(3)$ & $0.9226(3)$ & $0.0094(2)$ & $0.0344(6)$ \\
C8 & $0.7976(4)$ & $1.0622(3)$ & $-0.0367(3)$ & $0.0511(8)$ \\
H8A & 0.730469 & 1.136625 & -0.049832 & $0.077^{*}$ \\
H8B & 0.812386 & 1.038609 & -0.105307 & $0.077^{*}$ \\
H8C & 0.875616 & 1.101212 & 0.015278 & $0.077^{*}$ \\
C9 & $0.6338(4)$ & $0.8624(4)$ & $-0.0654(3)$ & $0.0559(9)$ \\
H9A & 0.611252 & 0.772368 & -0.033698 & $0.084^{*}$ \\
H9B & 0.642508 & 0.839113 & -0.136337 & $0.084^{*}$ \\
H9C & 0.567195 & 0.936832 & -0.074587 & $0.084^{*}$
\end{tabular}




$\begin{array}{lllll}\mathrm{C} 10 & 0.8637(3) & 0.8100(4) & 0.0377(3) & 0.0506(8) \\ \mathrm{H} 10 \mathrm{~A} & 0.936443 & 0.849222 & 0.095807 & 0.076^{*} \\ \mathrm{H} 10 \mathrm{~B} & 0.888703 & 0.789497 & -0.02666 & 0.076^{*} \\ \mathrm{H} 10 \mathrm{C} & 0.834626 & 0.718248 & 0.062297 & 0.076^{*} \\ \mathrm{~N} 1 & 0.7077(2) & 0.9636(2) & 0.26832(16) & 0.0263(4) \\ \mathrm{H} 1 & 0.725256 & 1.058913 & 0.272455 & 0.032^{*} \\ \text { O1 } & 0.73962(17) & 0.97953(18) & 0.11063(13) & 0.0272(4) \\ \text { O2 } & 0.69111(17) & 0.75519(18) & 0.16743(14) & 0.0289(4) \\ \mathrm{I} 1 & 0.04221(2) & 1.09875(2) & 0.33737(2) & 0.03907(7)\end{array}$

Atomic displacement parameters $\left(\AA^{2}\right)$

\begin{tabular}{lllllll}
\hline & $U^{11}$ & $U^{22}$ & $U^{33}$ & $U^{12}$ & $U^{13}$ & $U^{23}$ \\
\hline C1 & $0.0294(14)$ & $0.0398(14)$ & $0.0301(13)$ & $0.0020(11)$ & $0.0106(10)$ & $0.0027(11)$ \\
C2 & $0.0333(14)$ & $0.0328(13)$ & $0.0263(12)$ & $-0.0012(11)$ & $0.0110(10)$ & $0.0031(10)$ \\
C3 & $0.0312(14)$ & $0.0309(13)$ & $0.0250(11)$ & $-0.0015(10)$ & $0.0139(10)$ & $0.0011(10)$ \\
C4 & $0.0337(14)$ & $0.0284(12)$ & $0.0236(11)$ & $-0.0036(10)$ & $0.0135(10)$ & $0.0005(9)$ \\
C5 & $0.0298(13)$ & $0.0321(13)$ & $0.0259(12)$ & $0.0007(10)$ & $0.0132(10)$ & $0.0032(10)$ \\
C6 & $0.0180(11)$ & $0.0216(11)$ & $0.0271(11)$ & $0.0013(8)$ & $0.0087(9)$ & $0.0020(9)$ \\
C7 & $0.0490(17)$ & $0.0323(13)$ & $0.0293(13)$ & $-0.0017(12)$ & $0.0229(12)$ & $-0.0034(10)$ \\
C8 & $0.085(3)$ & $0.0407(17)$ & $0.0437(17)$ & $-0.0055(16)$ & $0.0430(18)$ & $0.0016(13)$ \\
C9 & $0.067(2)$ & $0.065(2)$ & $0.0325(15)$ & $-0.0141(18)$ & $0.0106(15)$ & $-0.0046(15)$ \\
C10 & $0.065(2)$ & $0.0415(17)$ & $0.063(2)$ & $0.0074(15)$ & $0.0446(18)$ & $-0.0051(15)$ \\
N1 & $0.0334(11)$ & $0.0215(10)$ & $0.0304(10)$ & $-0.0039(8)$ & $0.0191(9)$ & $-0.0015(8)$ \\
O1 & $0.0396(10)$ & $0.0196(8)$ & $0.0293(9)$ & $0.0004(7)$ & $0.0209(7)$ & $0.0004(7)$ \\
O2 & $0.0348(10)$ & $0.0197(8)$ & $0.0352(9)$ & $-0.0030(7)$ & $0.0157(8)$ & $-0.0001(7)$ \\
I1 & $0.02499(10)$ & $0.04928(12)$ & $0.04157(11)$ & $0.00657(8)$ & $0.00878(7)$ & $0.00614(8)$ \\
& & & & & &
\end{tabular}

Geometric parameters $\left(\AA,^{\circ}\right)$

\begin{tabular}{llll}
\hline $\mathrm{C} 1-\mathrm{C} 2$ & $1.193(4)$ & $\mathrm{C} 7-\mathrm{C} 9$ & $1.514(4)$ \\
$\mathrm{C} 1-\mathrm{I} 1$ & $1.999(3)$ & $\mathrm{C} 7-\mathrm{C} 8$ & $1.521(4)$ \\
$\mathrm{C} 2-\mathrm{C} 3$ & $1.385(4)$ & $\mathrm{C} 8-\mathrm{H} 8 \mathrm{~A}$ & 0.98 \\
$\mathrm{C} 3-\mathrm{C} 4$ & $1.191(4)$ & $\mathrm{C} 8-\mathrm{H} 8 \mathrm{~B}$ & 0.98 \\
$\mathrm{C} 4-\mathrm{C} 5$ & $1.472(3)$ & $\mathrm{C} 8-\mathrm{H} 8 \mathrm{C}$ & 0.98 \\
$\mathrm{C} 5-\mathrm{N} 1$ & $1.452(3)$ & $\mathrm{C} 9-\mathrm{H} 9 \mathrm{~A}$ & 0.98 \\
$\mathrm{C} 5-\mathrm{H} 5 \mathrm{~A}$ & 0.99 & $\mathrm{C} 9-\mathrm{H} 9 \mathrm{~B}$ & 0.98 \\
$\mathrm{C} 5-\mathrm{H} 5 \mathrm{~B}$ & 0.99 & $\mathrm{C} 9-\mathrm{H} 9 \mathrm{C}$ & 0.98 \\
$\mathrm{C} 6-\mathrm{O} 2$ & $1.223(3)$ & $\mathrm{C} 10-\mathrm{H} 10 \mathrm{~A}$ & 0.98 \\
$\mathrm{C} 6-\mathrm{O} 1$ & $1.338(3)$ & $\mathrm{C} 10-\mathrm{H} 10 \mathrm{~B}$ & 0.98 \\
$\mathrm{C} 6-\mathrm{N} 1$ & $1.340(3)$ & $\mathrm{C} 10-\mathrm{H} 10 \mathrm{C}$ & 0.98 \\
$\mathrm{C} 7-\mathrm{O} 1$ & $1.486(3)$ & $\mathrm{N} 1-\mathrm{H} 1$ & 0.88 \\
$\mathrm{C} 7-\mathrm{C} 10$ & $1.512(4)$ & & 109.5 \\
$\mathrm{C} 2-\mathrm{C} 1-\mathrm{I} 1$ & & & 109.5 \\
$\mathrm{C} 1-\mathrm{C} 2-\mathrm{C} 3$ & $177.3(3)$ & $\mathrm{H} 8 \mathrm{~A}-\mathrm{C} 8-\mathrm{H} 8 \mathrm{~B}$ & 109.5 \\
$\mathrm{C} 4-\mathrm{C} 3-\mathrm{C} 2$ & $179.1(3)$ & $\mathrm{C} 7-\mathrm{C} 8-\mathrm{H} 8 \mathrm{C}$ & 109.5 \\
$\mathrm{C} 3-\mathrm{C} 4-\mathrm{C} 5$ & $179.4(3)$ & $\mathrm{H} 8 \mathrm{~A}-\mathrm{C} 8-\mathrm{H} 8 \mathrm{C}$ &
\end{tabular}




$\begin{array}{llll}\mathrm{N} 1-\mathrm{C} 5-\mathrm{C} 4 & 112.5(2) & \mathrm{C} 7-\mathrm{C} 9-\mathrm{H} 9 \mathrm{~A} & 109.5 \\ \mathrm{~N} 1-\mathrm{C} 5-\mathrm{H} 5 \mathrm{~A} & 109.1 & \mathrm{C} 7-\mathrm{C} 9-\mathrm{H} 9 \mathrm{~B} & 109.5 \\ \mathrm{C} 4-\mathrm{C} 5-\mathrm{H} 5 \mathrm{~A} & 109.1 & \mathrm{H} 9 \mathrm{~A}-\mathrm{C} 9-\mathrm{H} 9 \mathrm{~B} & 109.5 \\ \mathrm{~N} 1-\mathrm{C} 5-\mathrm{H} 5 \mathrm{~B} & 109.1 & \mathrm{H} 9 \mathrm{~A}-\mathrm{C} 9-\mathrm{H} 9 \mathrm{H} 9 \mathrm{C} & 109.5 \\ \mathrm{C} 4-\mathrm{C} 5-\mathrm{H} 5 \mathrm{~B} & 109.1 & \mathrm{H} 9 \mathrm{~B}-\mathrm{C} 9-\mathrm{H} 9 \mathrm{C} & 109.5 \\ \mathrm{H} 5 \mathrm{~A}-\mathrm{C} 5-\mathrm{H} 5 \mathrm{~B} & 107.8 & \mathrm{C}-\mathrm{C} 10-\mathrm{H} 10 \mathrm{~A} & 109.5 \\ \mathrm{O} 2-\mathrm{C} 6-\mathrm{O} 1 & 125.7(2) & \mathrm{H} 10 \mathrm{C}-\mathrm{C} 10-\mathrm{H} 10 \mathrm{~B} & 109.5 \\ \mathrm{O} 2-\mathrm{C} 6-\mathrm{N} 10 \mathrm{~B} & 109.5 \\ \mathrm{O} 1-\mathrm{C} 6-\mathrm{N} 1 & 124.3(2) & \mathrm{C}-\mathrm{C} 10-\mathrm{H} 10 \mathrm{C} & 109.5 \\ \mathrm{O} 1-\mathrm{C} 7-\mathrm{C} 10 & 110.00(19) & \mathrm{H} 10 \mathrm{~A}-\mathrm{C} 10-\mathrm{H} 10 \mathrm{C} & 109.5 \\ \mathrm{O} 1-\mathrm{C} 7-\mathrm{C} 9 & 109.6(2) & \mathrm{C} 6-\mathrm{N} 1-\mathrm{C} 5 & 109.5 \\ \mathrm{C} 10-\mathrm{C} 7-\mathrm{C} 9 & 109.6(2) & \mathrm{C} 6-\mathrm{N} 1-\mathrm{H} 1 & 109.5 \\ \mathrm{O} 1-\mathrm{C} 7-\mathrm{C} 8 & 113.4(3) & \mathrm{C} 5-\mathrm{N} 1-\mathrm{H} 1 & 122.3(2) \\ \mathrm{C} 10-\mathrm{C} 7-\mathrm{C} 8 & 101.7(2) & \mathrm{C} 6-\mathrm{O} 1-\mathrm{C} 7 & 118.8 \\ \mathrm{C} 9-\mathrm{C} 7-\mathrm{C} 8 & 110.5(3) & & 118.8 \\ \mathrm{C} 7-\mathrm{C} 8-\mathrm{H} 8 \mathrm{~A} & 111.5(3) & \mathrm{N} 1-\mathrm{C} 6-\mathrm{O} 1-\mathrm{C} 7 & 121.17(18) \\ \mathrm{C} 7-\mathrm{C} 8-\mathrm{H} 8 \mathrm{~B} & 109.5 & \mathrm{C} 10-\mathrm{C} 7-\mathrm{O} 1-\mathrm{C} 6 & 175.9(2) \\ \mathrm{O} 2-\mathrm{C} 6-\mathrm{N} 1-\mathrm{C} 5 & 109.5 & \mathrm{C} 9-\mathrm{C} 7-\mathrm{O} 1-\mathrm{C} 6 & -58.9(3) \\ \mathrm{O} 1-\mathrm{C} 6-\mathrm{N} 1-\mathrm{C} 5 & & \mathrm{C} 8-\mathrm{C} 7-\mathrm{O} 1-\mathrm{C} 6 & 66.1(3) \\ \mathrm{C} 4-\mathrm{C} 5-\mathrm{N} 1-\mathrm{C} 6 & & & -175.8(2) \\ \mathrm{O} 2-\mathrm{C} 6-\mathrm{O} 1-\mathrm{C} 7 & 178.5(2) & & \end{array}$

Hydrogen-bond geometry $\left(\AA,{ }^{\circ}\right)$

\begin{tabular}{lllll}
\hline$D-\mathrm{H} \cdots A$ & $D-\mathrm{H}$ & $\mathrm{H} \cdots A$ & $D \cdots A$ & $D-\mathrm{H} \cdots A$ \\
\hline $\mathrm{N} 1-\mathrm{H} 1 \cdots \mathrm{O} 2^{\mathrm{i}}$ & 0.88 & 2.04 & 2.881 & 160 \\
$\mathrm{C} 1-\mathrm{I} 1 \cdots \mathrm{O} 2^{\mathrm{ii}}$ & 2.00 & 2.95 & 4.919 & 168
\end{tabular}

Symmetry codes: (i) $-x+3 / 2, y+3 / 2,-z+3 / 2$; (ii) $-x+1 / 2, y+3 / 2,-z+3 / 2$. 